\title{
Alfabetização financeira dos universitários lusófonos: Evidências de uma universidade do interior do Ceará, Brasil
}

Financial literacy of lusophone university students: Evidence from a university in the interior of

\author{
Ceará, Brazil
}

Alfabetización financiera de estudiantes universitarios de habla portuguesa: Evidencia de una universidad en el interior de Ceará, Brasil

Recebido: 24/06/2021 | Revisado: 01/07/2021 | Aceito: 15/07/2021 | Publicado: 24/07/2021

\author{
Sâmia Nagib Maluf \\ ORCID: https://orcid.org/0000-0001-8319-555X \\ Universidade da Integração Internacional da Lusofonia Afro-Brasileira, Brasil \\ E-mail: snmaluf@gmail.com \\ Ana Gabrielly Morais Silva \\ ORCID: https://orcid.org/0000-0002-7227-3898 \\ Universidade da Integração Internacional da Lusofonia Afro-Brasileira, Brasil \\ E-mail: gabriellymorais29@gmail.com \\ Breno Cândido Cordeiro \\ ORCID: https://orcid.org/0000-0003-3537-0370 \\ Universidade da Integração Internacional da Lusofonia Afro-Brasileira, Brasil \\ E-mail: brenoccordeiro@gmail.com
}

\begin{abstract}
Resumo
Os auxílios financeiros são fornecidos aos discentes, mas como toda e qualquer política deve ser acompanhada e avaliada. Para estabelecer o perfil, hábitos e a forma como esses recursos são geridos, torna-se necessário conhecer o grau de alfabetização financeira dos beneficiários. Calculou-se o índice de alfabetização financeira de 211 discentes (nacionais e estrangeiros lusófonos, dos Campi da Universidade da Integração Internacional da Lusofonia AfroBrasileira-Unilab, interior do Ceará), representando 13,6\% dos beneficiários de 2018. Estudo de caso quantitativo e descritivo. A metodologia para a construção do questionário e cálculo dos índices baseou-se na OCDE/INFE (2018). Investigou-se as possíveis influências das variáveis socioeconômicas e demográficas sobre a alfabetização através da Modelagem Linear Automático, utilizando todos os subconjuntos dos possíveis preditores do modelo; após assegurada a normalidade (Shapiro-Wilk) e ausência de correlações (Pearson). Encontrou-se 0.68 para o comportamento, 0.41 para atitude e 0.23 para o conhecimento financeiro, considerados baixos, em especial, o conhecimento financeiro. As variáveis que mais afetaram os resultados foram as de curso, religião e idade. Em conclusão, o estudo sugere que esforços devam ser empreendidos em programas de educação financeira continuados.

Palavras-chave: Alfabetização financeira; Conhecimento financeiro; Atitude financeira; Comportamento financeiro; Universitários lusófonos.
\end{abstract}

\begin{abstract}
Financial aid is provided to students, but like any policy it must be monitored and evaluated. To establish the profile, habits and the way these resources are managed, it is necessary to know the degree of financial literacy of the beneficiaries. The financial literacy rate of 211 students (Portuguese-speaking nationals and foreigners, from Unilab campuses, in the interior of Ceará), was calculated, representing 10.5\% of the beneficiaries in 2018. Quantitative and descriptive case study. The methodology for constructing the questionnaire and calculating the indexes was based on the OECD/INFE (2018). The possible influences of socioeconomic and demographic variables on literacy were investigated through Automatic Linear Modeling, using all subsets of the possible predictors of the model; after ensuring normality (Shapiro-Wilk) and absence of correlations (Pearson). It was found 0.68 for behavior, 0.41 for attitude and 0.23 for financial knowledge, considered low, especially financial knowledge. The variables that most affected the results were course, religion and age. In conclusion, the study suggests that efforts should be made in continuing financial education programs.
\end{abstract}

Keywords: Financial literacy; Financial knowledge; Financial behavior; Financial attitude; Lusophone university students.

\section{Resumen}

Se proporciona ayuda financiera a los estudiantes, pero como cualquier política, debe ser monitoreada y evaluada. Para establecer el perfil, los hábitos y la forma en que se gestionan estos recursos, es necesario conocer el grado de 
alfabetización financiera de los beneficiarios. Se calculó la tasa de alfabetización financiera de 211 estudiantes (nacionales y extranjeros de habla portuguesa, de campus de Unilab, en el interior de Ceará), lo que representa el $13,6 \%$ de los beneficiarios en 2018. Estudio de caso cuantitativo y descriptivo. La metodología para la construcción del cuestionario y el cálculo de los índices se basó en la OCDE / INFE (2018). Las posibles influencias de las variables socioeconómicas y demográficas sobre la alfabetización se investigaron mediante el modelado lineal automático, utilizando todos los subconjuntos de los posibles predictores del modelo; después de asegurar la normalidad (Shapiro-Wilk) y la ausencia de correlaciones (Pearson). Se encontró 0.68 para comportamiento, 0.41 para actitud y 0.23 para conocimiento financiero, considerado bajo, especialmente conocimiento financiero. Las variables que más incidieron en los resultados fueron curso, religión y edad. En conclusión, el estudio sugiere que se deben realizar esfuerzos en los programas de educación financiera continua.

Palabras clave: Conocimientos financeiros; Actitud financeira; Comportamiento financeiro; Estudiantes universitarios de habla portuguesa.

\section{Introdução}

O mercado financeiro tem se sofisticado, ao longo do tempo, levando a que as famílias tomem decisões que poderão afetar não somente o seu bem-estar; mas também comprometer gerações futuras. A comunidade internacional vem estabelecendo e definindo vários compromissos com o intuito de fomentar o desenvolvimento de regiões carentes através da ampliação da oferta de cursos superiores e de redes de cooperação. Países em melhores condições de desenvolvimento têm sido conclamados a cooperar com aqueles que estejam em condições desfavoráveis, para que esses vençam obstáculos estruturais que impedem o desenvolvimento global

A educação financeira é um mecanismo importante para auxiliar as famílias a realizar o seu orçamento e gerenciar suas receitas de forma eficiente, além de evitar que sejam vítimas de fraudes e de manobras do sistema financeiro e dos agentes econômicos que possam, porventura, beneficiarem-se de alguma assimetria de informação. (Cenci, Pereira \& Barichello, 2015; Lima, Barbalho, Nascimento, Medeiros Neto \& Brito, 2021; OECD, 2005; Reis, Borgis \& Silva, 2021; Santos, Rodrigues \& Nunes, 2021; Seixas, Santarosa \& Ferrão, 2020).

A Organização para a Cooperação e Desenvolvimento Econômico (OCDE/OECD) ${ }^{1}$ foi criada com a finalidade de obter evidências baseadas em padrões internacionais e encontrar soluções para os vastos desafios sociais econômicos e ambientais. Em julho de 2005, recomendou a promoção da educação e conscientização financeira; através da disseminação dos princípios e boas práticas entre instituições públicas e privadas (com ou sem fins lucrativos), envolvidas em educação e conscientização financeira. A crise mundial de 2008 evidenciou o despreparado dos consumidores em relação aos conhecimentos financeiros e confirmou as recomendações realizadas. A OECD/INFE ${ }^{2}$ foi instituída para realizar desenho, acompanhar a implementação e avaliar impacto de políticas públicas relativos à educação financeira. Nessa perspectiva, criaram um kit de ferramentas para coleta de dados concernentes à alfabetização financeira, dado que essa metodologia permite comparação internacional. E é nessa perspectiva que esse estudo se insere.

A educação financeira busca desenvolver nas pessoas habilidades para melhorar suas tomadas de decisões, levando a que os indivíduos considerem todas as alternativas disponíveis, explorem oportunidades e atinjam seus objetivos pessoais. A importância da educação financeira pode ser vista tanto sob a perspectiva do bem-estar pessoal quanto da sociedade. Indivíduos desorganizados financeiramente poderão prejudicar não somente o seu consumo, mas a sua carreira; assim como comprometer gerações futuras. E quando a desorganização dos indivíduos se amplia, pode-se chegar a casos extremos de comprometimento de determinados mercados, podendo chegar até a demandar interferência governamental, através de políticas públicas de correção, que em última instância implicam em custos para a administração pública (Atkinson \& Messy,

\footnotetext{
${ }^{1}$ Convenção assinada em 14/12/1960, entrou em vigor após 30/09/1961; sucedeu a OECE (1948). O Brasil tornou-se um país parceiro, a partir de 1990. https://www.oecd.org/latin-america/paises/brasil- https://www.oecd.org/global-relations/keypartners/\#d.en.194387.

${ }^{2}$ https://www.oecd.org/financial/education/oecd-international-network-on-financial-education.html.
} 
2012; Bernheim, Garrett \& Maki, 1997; Holzmann \& Miralles, 2005; Jacob \& Busch, 2000; Menezhetti Neto, Falcetta, Rassier \& Marchionatti, 2014).

O acesso da população brasileira ao ensino superior avançou bastante nos últimos anos, apesar de que na perspectiva da OCDE, o Brasil ainda tem um longo caminho a percorrer. Segundo Barros (2015) a taxa de escolarização líquida da população brasileira de 18 a 24 anos, de acordo com o Censo da Educação Superior, (INEP, 2010) era da ordem de 14,4; enquanto que a PNAD contínua (IBGE, 2019) demonstra que, em 2018, a taxa de escolarização das pessoas de 18 a 24 anos, considerando todos os cursos, foi de $32.7 \%$; entretanto, somente $25.2 \%$ frequentavam cursos da educação superior ou haviam completado esse nível.

Nos últimos anos, o Brasil tem realizado importantes parcerias no âmbito da Cooperação Sul-Sul rumo à concretização deste objetivo, principalmente com aqueles países com baixo Índice de Desenvolvimento Humano (IDH), destacando-se os Países Africanos de Língua Oficial Portuguesa (PALOP) - Angola, Cabo Verde, Guiné Bissau, Guiné Equatorial, Moçambique e São Tomé e Príncipe. A Universidade da Integração Internacional da Lusofonia Afro-Brasileira (Unilab) foi criada pela Lei 12.289/2010, iniciou as suas atividades acadêmicas em maio de 2011 e nasceu nesta perspectiva de interiorização e internacionalização do ensino superior e oferta vagas tanto para nacionais quanto para os estrangeiros de países lusófonos, em especial da África.

$\mathrm{Se}$, de um lado, ainda existe uma pequena parcela que tem acesso ao ensino superior, por outro lado, espera-se que essa escolarização propicie e forme pessoas que consigam tomar decisões financeiras mais assertivamente daquelas que não tem acesso ao ensino superior. E, como a alfabetização financeira contribui para geração de bem estar tanto para a geração atual, quanto para a geração futura; surgiu a seguinte pergunta de qual é o índice de alfabetização dos universitários dos países lusófonos dos Campi do Ceará, da Unilab?

Considerando a relevância do tema e a inexistência de estudos relativos à alfabetização financeira dos discentes da Unilab, a pesquisa foi realizada junto aos universitários (nacionais e estrangeiros dos países lusófonos) que receberam benefícios em 2018, dos Campi do interior do Ceará, da Unilab. O trabalho apresenta primeiramente a alfabetização financeira posteriormente discorre sobre a metodologia, apresenta e discute os dados e finaliza com as considerações finais.

\section{Alfabetização Financeira}

\subsection{Introdução}

A combinação de baixo índice de desenvolvimento humano com alto grau de concentração de renda resulta em um mercado extremamente vulnerável a toda e qualquer oscilação econômica. E, países com grandes desigualdades nas distribuições das rendas, como é o caso do Brasil e dos países africanos lusófonos necessita-se que a população maximize a utilização da sua renda, através do consumo consciente e do planejamento orçamentário; além de reduzir o nível do seu endividamento (OECD 2005, OECD/INFE 2016; 2020).

Segundo Bernheim, Garrett e Maki (1997), o processo de Educação Financeira está mais avançado nos países desenvolvidos; tendo-se tornado, em alguns deles, obrigatória nas escolas secundárias. A assimetria de informações em relação à alocação da renda afeta desigualmente aqueles que dela mais necessitam - a população carente -, principalmente nos países em desenvolvimento. E essa assimetria pode ser reduzida através da educação, no caso, a financeira.

A OCDE tem protagonizado e coordenado vários programas $^{3}$ e estudos com vistas a avaliar as políticas e resultados educacionais concernentes à educação financeira. A OECD (2005) estabeleceu princípios e as boas práticas de educação

\footnotetext{
${ }^{3}$ Implementação e coordenação do Programa Internacional de Avaliação de Estudantes (PISA), rede mundial que avalia o desempenho escolar desde 2000, que permite comparação internacional dos dados, a cada dois anos, com vistas a melhorar as políticas e resultados educacionais (leitura, matemática e ciências - em todas as edições e ciclos; além de resolução de problemas, letramento financeiro e
} 
financeira (sugeriu ações públicas, indicou formas e o papel das instituições financeiras, formas de como trabalhar a educação financeira para poupança de aposentadoria e o desenvolvimento de programas de educação financeira) para conscientização financeira. Consideram a educação financeira pode ser definida como:

o processo pelo qual consumidores/investidores financeiros aprimoram sua compreensão sobre produtos, conceitos e riscos financeiros e, por meio de informação, instrução e/ou aconselhamento objetivo, desenvolvam as habilidades e a confiança para se tornarem mais conscientes de riscos e oportunidades financeiras, a afazer escolhas informadas, a saber onde buscar ajuda, e a tomar outras medidas efetivas para melhorar seu bem-estar financeiro (OCDE, 2005, Anexo).

Na perspectiva brasileira, como política de Estado, em 2010, estabeleceu-se a Estratégia Nacional de Educação Financeira (ENEF) e, em $2020^{4}$, foi instituída uma nova estratégia além do Fórum Brasileiro de Educação Financeira (FBEF) ${ }^{5}$, órgão colegiado de articulação com a competência para implementar e estabelecer os princípios da ENEF, além de divulgar, compartilhar e promover a interlocução das ações de educação financeira, securitária, previdenciária e fiscal.

Huston (2010) identificou a utilização dos termos de alfabetização financeira, conhecimento financeiro e educação financeira como sinônimos, tanto pela mídia quanto pela academia. Baseado na sugestão de Marcolin e Abraham (2006, apud Huston, 2010) de exploração e desenvolvimento da literatura e em especial, para forma de medir a alfabetização financeira de forma padronizada e que fosse passível de comparação internacional.

Entretanto, fez-se necessário primeiramente a concepção e definição de constructos de alfabetização financeira para, posteriormente, definir o conteúdo do instrumento e a forma de interpretar a medida.

\subsection{Conceito}

Partindo de um conceito mais amplo da alfabetização baseado no entendimento e no uso de toda e qualquer informação quantitativa, chegou-se à congruência de que tanto o conceito quanto a concepção da alfabetização financeira e da educação financeira são constructos diferentes do capital humano. Nesse sentido, o conhecimento financeiro seria uma dimensão que faria parte da alfabetização financeira que, por sua vez, teria uma dimensão mais ampla, na medida em que o indivíduo deveria ter a habilidade e confiança para tomar as suas decisões financeiras. A Figura 1 ilustra essas conceituações de Borges e Botelho (2020), com base nos conceitos da OECD/INFE (2013).

competência). O PISA tem por objetivo avaliar a habilidade dos estudantes na faixa etária de 15 anos (com término da escolaridade básica) para aplicar os seus conhecimentos na resolução de problemas e decisões financeiras, contextualizando em situações reais. Em 2018, 79 países participaram do PISA ( 37 da OCDE e 42 de países/economias parceiras).

${ }^{4}$ O Decreto 7.397 de 22/12/2010 criou a ENEF e o Decreto 10.393 de 09/06/2020 revogou o decreto anterior e instituiu a nova Estratégia Nacional de Educação Financeira.

${ }^{5}$ Integrantes: Banco Central, Comissão de Valores Mobiliários, Superintendência de Seguros Privados, Secretaria do Tesouro Nacional da Secretaria Especial de Fazenda do Ministério da Economia, Secretaria da Previdência da Secretaria Especial de Previdência e Trabalho do Ministério da Economia, Superintendência Nacional de Previdência Complementar, Secretaria Nacional do Consumidor do Ministério da Justiça e Segurança Pública e Ministério da Educação. 
Figura 1 - Educação Financeira versus alfabetização financeira.
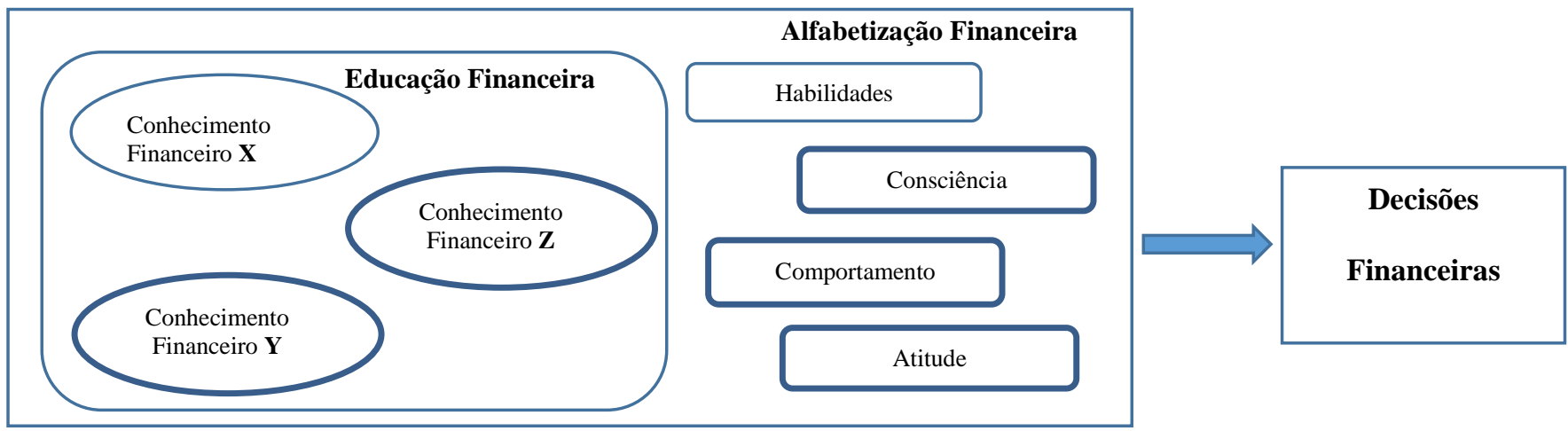

Fonte: Borges e Botelho (2020)

Anteriormente, Hung, Parker e Yoong (2009) consideraram quatro variáveis para compor a alfabetização financeira: conhecimento financeiro, atitude financeira, comportamento financeiro e habilidade financeira.

Já na revisão teórica de Remund (2010), no período de 2000 a 2010, concluiu que, apesar de não existir uniformidade nas definições para alfabetização financeira, encontrou categorias em comuns nos estudos, quais sejam: entendimento dos conceitos financeiros, atitude no gerenciamento das finanças pessoais, habilidade na tomada das decisões financeiras e segurança no planejamento do consumo futuro.

A OECD/INFE (2010), na tentativa de desenvolver uma medida que fosse padrão e comparável iniciou estudos através da aplicação de questionários, com base em três constructos que iriam compor a alfabetização financeira, quais sejam: comportamento financeiro, atitude e conhecimento financeiro.

Na perspectiva conceitual, a OECD considera que a alfabetização financeira compreende a conjugação de consciência, conhecimento, habilidade, atitude e comportamento na tomada das decisões financeiras, com vistas a buscar o bem-estar financeiro individual (Atkinson \& Messy, 2012).

Nessa perspectiva o Quadro 1 apresenta os conceitos, dimensões e os autores relativos à alfabetização financeira.

Quadro 1 - Principais conceitos e dimensões que envolvam a alfabetização financeira.

\begin{tabular}{|c|c|c|}
\hline Conceitos de Alfabetização Financeira & Dimensões & Autores \\
\hline $\begin{array}{l}\text { O conhecimento financeiro e a aplicação desse conhecimento, com } \\
\text { autoconfiança na tomada de decisões financeiras. }\end{array}$ & $\begin{array}{l}\text { Conhecimento financeiro e } \\
\text { aplicação do conhecimento. }\end{array}$ & Huston (2010) \\
\hline $\begin{array}{l}\text { A capacidade de usar o conhecimento e as habilidades adquiridas para } \\
\text { uma melhor gestão. }\end{array}$ & $\begin{array}{l}\text { Conhecimento financeiro e } \\
\text { habilidades. }\end{array}$ & Hung, Parker e Yoong (2009) \\
\hline $\begin{array}{l}\text { A capacidade de tomar decisões simples no contexto cotidiano das } \\
\text { escolhas financeiras. }\end{array}$ & Decisões cotidianas. & Lusardi e Tufano (2009). \\
\hline $\begin{array}{l}\text { A capacidade de compreender a informação financeira e tomar } \\
\text { decisões eficazes, utilizando essa informação. }\end{array}$ & Compreensão e decisão. & $\begin{array}{l}\text { Servon e Kaestner (2008); Robb } \\
\text {, Babiarz e Woodyard (2012) }\end{array}$ \\
\hline $\begin{array}{l}\text { Vai além da idéia básica da educação financeira, onde a influência do } \\
\text { conhecimento financeiro é medida pelas atitudes financeiras. }\end{array}$ & Compreensão e decisão. & $\begin{array}{l}\text { Servon e Kaestner (2008); } \\
\text { Robb, Babiarz e Woodyard } \\
(2012) \text {. }\end{array}$ \\
\hline $\begin{array}{l}\text { A escolha de inúmeras alternativas para o estabelecimento dos } \\
\text { objetivos financeiros. }\end{array}$ & Escolha eficaz & Criddle (2006) \\
\hline A tomada de decisões financeiras informadas. & Decisões financeiras & Redmund (2010) \\
\hline $\begin{array}{l}\text { O capital humano mais específico, medido através de questões de } \\
\text { conhecimentos financeiros. }\end{array}$ & Conhecimento financeiro. & $\begin{array}{l}\text { Lusardi e Tufano (2009); Robb } \\
\text { e Sharpe (2009). }\end{array}$ \\
\hline $\begin{array}{l}\text { Mensurada através de um conjunto de perguntas que medem conceitos } \\
\text { financeiros básicos, tais como capitalização de juros, inflação e } \\
\text { diversificação de risco. }\end{array}$ & Conhecimento financeiro. & Lusardi e Mitchell (2013). \\
\hline $\begin{array}{l}\text { Engloba a alfabetização financeira três dimensões: o conhecimento } \\
\text { financeiro, o comportamento financeiro e a atitude financeira. }\end{array}$ & $\begin{array}{l}\text { Conhecimento financeiro, } \\
\text { comportamento financeiro e } \\
\text { atitude financeira. }\end{array}$ & $\begin{array}{l}\text { Atkinson e Messy (2012); } \\
\text { OECD (2013). }\end{array}$ \\
\hline
\end{tabular}


Esse estudo baseia-se na conceituação de Atkinson e Messy (2012) e da OECD (2013).

\subsection{Estudos realizados e principais resultados}

Em termos internacionais, resultados de baixo nível de conhecimento financeiro foram encontrados nos estudos de Ansong (2011) ao investigar o conhecimento financeiro de 343 universitários de Ghana (Univsersity of Cape Coast). Sekita (2011), encontrou que o nível de alfabetização financeira não é alto, no Japão. Muitos respondentes não responderam as questões relativas à alfabetização financeira, indicando que insegurança. Mulheres, jovens e a população com baixa renda e baixo nível educacional obtiveram os menos níveis de alfabetização financeira. Conclui que a alfabetização aumenta a probabilidade de ter um plano de poupança para a aposentadoria. Agarwalla, Barua, Jacob e Varma (2012) encontraram um baixo conhecimento financeiro dos indianos, se comparado com os padrões internacionais de Lusardi, Mitchel e Curto (2010), nos EUA.

No âmbito dos estudos nacionais, independentemente do tipo de metodologia utilizada e do público-alvo, os resultados convergem para o baixo nível do conhecimento financeiro. Lucci, Zerremer, Verrone e Santos (2006) encontraram que o nível dos conceitos financeiros está correlacionado positivamente com o nível de educação financeira, ao investigaram 122 universitários (Administração e Ciências Contábeis), da Faculdade Independente Butantã. Poltrich, Vieira e Ceretta (2013) encontraram, em uma amostra de 534 universitários do Rio Grande do Sul, baixos níveis de conhecimento financeiro (básico: 0.436; avançado: 1.034 e geral: 1.470). Poltrich, Vieira e Kirch (2014), buscaram desenvolver um indicador para a alfabetização (Termômetro de Alfabetização Financeira), utilizando clusters para uma amostra de 1.572 habitantes adultos (acima de 18 anos) do Rio Grande do Sul e os resultados encontrados para os dois níveis de alfabetização (baixo: 0.57 e alto: 0.82) foram considerados preocupantes, dado que $67.9 \%$ da amostra apresentou baixo nível de conhecimento financeiro. Jobim e Losekann (2015) aplicaram 126 questionários aos universitários da Universidade da Região da Campanha (São Gabriel, Rio Grande do Sul), fazendo uso da Análise Fatorial Exploratória (AFE), encontraram um baixo nível de alfabetização financeira (37\% de desempenho). Silva, Rodrigues, Moura e Castro (2019) aplicaram 395 questionários (amostra de 50\%) junto aos universitários de uma instituição de ensino superior de Divinópolis, Minas Gerais e não encontraram evidências da influência do período de estudo dos universitários sobre o nível de conhecimento financeiro, sejam para os discentes dos primeiros ou dos últimos períodos. Zuliani, Flores, Floriano, Josende e Ramos (2020) estudaram uma amostra de 64, de uma população de 174 servidores militares de uma organização militar da fronteira gaúcha e encontraram que, a maior parte do efetivo apresentou baixo nível de alfabetização financeira, dado que $61 \%$ dos respondentes apresentaram, em média uma pontuação de 0.35 para atitude financeira, 0.52 para o comportamento financeiro e 0.49 para o conhecimento financeiro.

Em relação ao último estudo em que o Brasil foi contemplado, na OECD/INFE (2016), utilizando a metodologia de 2015, encontraram as médias de 4.3 para o conhecimento financeiro, 4.6 para o comportamento financeiro e de 3.1 para a atitude financeira.

Na perspectiva da construção de uma medida, o desenvolvimento tem sido mais complexos e pode-se classificar em dois tipos basicamente: os que seguem a metodologia da OECD/INFE (2010, 2013, 2016 e 2020) e os demais seguindo a literatura de Lusardi e Mitchell (2011), ressaltando os de Poltrich, Vieira e Kirch (2014) e o de Vieira, Moreira Júnior e Poltrich (2019).

Para além do cálculo do nível de alfabetização financeira, vários estudos investigaram variáveis socioeconômicas e demográficas que pudessem explicar o resultado encontrado para esses níveis de alfabetização financeira. O Quadro 2 apresenta alguns dos principais resultados dos estudos realizados. 
Quadro 2 - Síntese da relação entre as variáveis demográficas e socioeconômicas e a alfabetização financeira.

\begin{tabular}{|c|c|c|}
\hline Variáveis & Relação com a Alfabetização Financeira & Autores \\
\hline Gênero & $\begin{array}{l}\text { - As mulheres geralmente apresentam menores índices de alfabetização financeira. } \\
\text { - As mulheres são menos propensas a responder às perguntas corretamente e mais } \\
\text { propensas a dizer que não sabem a resposta. } \\
\text { - A educação financeira dos homens está aumentando mais rapidamente do que das } \\
\text { mulheres. } \\
\text { - Fazendo um comparativo entre mulheres, aquelas casadas e com renda mais alta } \\
\text { possuem melhores níveis de alfabetização financeira. }\end{array}$ & $\begin{array}{l}\text { Chen e Volpe (1998) } \\
\text { Lusardi e Mitchell (2005) } \\
\text { Lusardi e Mitchell (2011) } \\
\text { Atkinson e Messy (2012) } \\
\text { Agarwalia } \text { et al. (2012) }\end{array}$ \\
\hline Idade & $\begin{array}{l}\text { - A idade média de } 30 \text { a } 40 \text { anos está associada com os maiores índices de } \\
\text { educação financeira. } \\
\text { - A educação financeira é mais baixa entre os mais jovens e mais velhos. }\end{array}$ & $\begin{array}{l}\text { Lusardi e Tufano (2009) } \\
\text { Lusardi e Mitchell (2011) } \\
\text { Finke } \text { et al. (2011) } \\
\text { Atkinson e Messy (2012) }\end{array}$ \\
\hline Estado civil & $\begin{array}{l}\text { - Os solteiros são significativamente mais propensos a ter menores conhecimentos } \\
\text { financeiros do que os casados. }\end{array}$ & Research (2003) \\
\hline Escolaridade & $\begin{array}{l}\text { - Aqueles com maiores níveis de alfabetização financeira são os que possuem } \\
\text { maiores níveis de escolaridade. } \\
\text { - O número de disciplinas ligadas à área financeira cursadas na graduação está } \\
\text { relacionado ao nível de educação financeira. } \\
\text { - Aqueles com menor nível educacional são menos propensos a responder às } \\
\text { perguntas corretamente e mais propensos a dizer que não sabem a resposta. }\end{array}$ & $\begin{array}{l}\text { Amadeu (2009) } \\
\text { Lusardi e Mitchell (2011) }\end{array}$ \\
\hline Renda & $\begin{array}{l}\text { - Baixos níveis de renda estão associados a baixos níveis de alfabetização } \\
\text { financeira. } \\
\text { - Alfabetização financeira e riqueza são conjuntamente determinadas e } \\
\text { correlacionadas ao longo do ciclo de vida. }\end{array}$ & $\begin{array}{l}\text { Lusardi e Tufano (2009) } \\
\text { Monticone (2010) } \\
\text { Jappelli e Padula (2011) } \\
\text { Hastings e Mitchell (2011) } \\
\text { Atkinson e Messy (2012) }\end{array}$ \\
\hline Trabalho & $\begin{array}{l}\text { - Indivíduos com maior tempo de serviço são mais alfabetizados financeiramente } \\
\text { em virtude da maior convivência com questões econômicas e financeiras, } \\
\text { enquanto que trabalhadores com baixa qualificação ou desempregados } \\
\text { apresentam atitudes e comportamentos menos desejáveis. }\end{array}$ & $\begin{array}{l}\text { Chen e Volpe (1988) } \\
\text { Research (2003) }\end{array}$ \\
\hline Etnia & $\begin{array}{l}\text { - Estudantes brancos apresentam melhores níveis de responsabilidade financeira. } \\
\text { - Negros e hispânicos são menos propensos a responder corretamente questões } \\
\text { sobre o futuro. . }\end{array}$ & $\begin{array}{l}\text { Lusardi e Mitchell (2005) } \\
\text { Grable e Joo (2006) } \\
\text { Lusardi e Mitchell (2011). }\end{array}$ \\
\hline
\end{tabular}

Fonte: Potrich, Vieira e Ceretta (2013).

Em termos de avaliação dos impactos dos programas de educação financeira, nomenclatura adotada por Lusardi, Michaud e Mitchell (2018), encontraram que os programas mais efetivos foram aqueles que proporcionavam acompanhamento e suporte de forma continuada aos indivíduos em relação aos conhecimentos adquiridos; dado que as pessoas em torno de 40 anos, podem aumentar a poupança em $10 \%$. Por outro lado, programas ministrados uma única vez produzem efeitos de curto prazo, mas com poucos efeitos no longo prazo.

\section{Metodologia}

O estudo enquadra-se como quantitativa, considerando a abordagem do problema. Por buscar identificar as características de populações ou fenômenos e de correlação entre variáveis, classifica-se como descrita; quanto aos objetivos. Por concentrar-se em um caso particular, classifica-se como estudo de caso. Os procedimentos técnicos utilizados foram a pesquisa bibliográfica e o levantamento. (Severino, 2017; Marconi e Lakatos, 2021). O plano amostral foi construído com base nos dados informados pela Pró-Reitoria de Políticas Afirmativas e Estudantis (Propae), da Unilab, em relação aos discentes nacionais e estrangeiros, de todos os cursos ofertados nos Campi do interior do Ceará (Auroras e Palmares), beneficiários do PAES, em 2018. Considerando a população de 1.549 discentes, a amostra foi selecionada de forma estratificada segundo gênero, curso (Administração Pública, Agronomia, Antropologia, BHU, Ciências Biológicas, Enfermagem, Engenharia de Energias, Física, História, Letras, Matemática, Pedagogia, Química e Sociologia) e nacionalidade (Brasil, Angola, Cabo Verde, Guiné-Bissau, São Tomé e Príncipe e Moçambique). A construção do questionário foi realizada seguindo o Kit de Ferramentas OECD/INFE (2018) para medir a Alfabetização Financeira e Inclusão Financeira, que tem por objetivo estabelecer uma 
metodologia que seja capaz que comparar os níveis de alfabetização e inclusão financeira entre diferentes países. No que se refere aos aspectos éticos, o projeto de pesquisa foi registrado na Plataforma Brasil, submetido e aprovado pelo Comitê de Ética em Pesquisa (CEP), da Unilab. Ressalta-se que os integrantes (autores) do estudo assinaram o Termo de Confidencialidade e de Responsabilidade da Pesquisa. Os dados foram obtidos por meio de questionário estruturado e pré testado, na parte inicial incluiu-se o Termo de Consentimento Livre e Esclarecido (TCLE), como requisito obrigatório para a validação das respostas. A coleta dos dados ocorreu no durante todo o mês de julho de 2020, através do Google Forms. A amostra considerada compreende os dados de 211 (duzentos e onze) questionários válidos, que representam 13,6\% da população, o que leva a considerá-la como representativa. Todos os procedimentos foram realizados no SPSS-26.

Ressalta-se que não existem resultados nacionais da OECD/INFE (2020) relativos à metodologia do Kit de Ferramentas de 2018, dado que o Brasil não foi avaliado neste estudo, somente no de 2016, com base na metodologia de 2015. Entretanto esse estudo baseou-se na metodologia da OECD/INFE (2018), em que, primeiramente calcula-se a pontuação de cada um dos constructos (atitude financeira, conhecimento financeiro e comportamento financeiro) e posteriormente é aferido o construto do índice de alfabetização financeira. O cálculo é computado através do número de respostas consideradas corretas e válidas para a pontuação. Para o caso do construto de conhecimento financeiro foram consideradas cinco questões, em decorrência do público-alvo. Dessa forma, a pontuação varia de 0 a 5 . O cálculo do comportamento financeiro também é computado através dos comportamentos definidos pela OECD/INFE (2018) como financeiramente inteligentes que variam entre 0 e 9. A pontuação das atitudes é calculada como a resposta média em três questões de atitude, variando de 1 a 5 . O cômputo do índice de alfabetização financeira é obtido através da soma das três pontuações mencionadas anteriormente, em que, considerando o questionário adaptado pode assumir qualquer valor entre 1 e 19. Para realizar a normalização, multiplicase por 100/19, conforme consta na Equação 1:

\section{$A l F_{i}=$ ConhecimentoFin $_{i} / 5+$ ComportamentoFin $_{i} / 9+$ AtitudeFin $_{i} / 5$}

Com vistas a investigar a existência de uma possível influência das variáveis socioeconômicas e demográficas sobre o grau de alfabetização, com base no Quadro 2, fez-necessário, primeiramente, realizar o diagnóstico da normalidade dos resíduos, através do Teste de Shapiro-Wilk. Posteriormente, realizou-se a análise da correlação baseado no Método de Pearson. Após confirmada a hipótese de normalidade e a inexistência de correlações, realizou-se a regressão, seguindo o procedimento de Modelagem Linear Automático (LINEAR) que para Yang (2013), utiliza todos os subconjuntos possíveis de preditores da variável dependente (VD) que devam ser incluídos no modelo, diferentemente do Método Stepwise que utiliza apenas um subconjunto de todos o espaço do modelo.

A Equação 2 apresenta o modelo estudado, em que a variável dependente é o índice de Alfabetização Financeira, obtido através da Equação 1. As variáveis explicativas ou independentes contempladas no estudo foram a Idade, Gênero, Estado Civil, Dependentes, Nacionalidade, Religião e Curso foram escolhidas com base no Quadro 2.

\section{$A l F_{i}=\alpha_{0}+\beta_{1} I$ Idade $i+\beta_{2} D_{1}$ gênero $_{i}+\beta_{3} D_{2}$ civili $_{i}+\beta_{4} D_{3}$ dependentes $_{i}+\beta_{5} D_{4}$ nacionalidade $_{i}+\beta_{6} D_{5}$ religião $_{i}+\beta_{7} D_{6}$ curso $_{i}+\varepsilon_{i}[2]$}

\section{Resultados e Discussão}

A apresentação dos resultados seguirá a sequência da metodologia, desta forma apresentar-se-á primeiramente os resultados relativos ao cálculo do índice de alfabetização, obtidos através da Equação 1, assim como a sua análise e discussão dos resultados. Posteriormente, serão apresentados os resultados tanto dos procedimentos e investigações necessárias para que 
a regressão seja realizada, segundo a Equação 2, assim como a respectiva análise e discussão dos resultados. A Tabela 1 apresenta a média dos resultados obtidos para a alfabetização financeira.

Tabela 1 - Índice do Alfabetização Financeira - Metodologia OCDE (2018) - Estatística Descritiva.

\begin{tabular}{l|l|l|l|l|l}
\hline Construtos & N & Mínimo & Máximo & Média & Erro Desvio \\
\hline Comportamento Financeiro & 211 & 3.00 & 9.00 & 6.8673 & 1.60992 \\
Atitude Financeira & 211 & 1.00 & 5.0 & 4.0783 & 1.06112 \\
Conhecimento Financeiro & 211 & .00 & 5 & 2.2701 & 1.40340 \\
Índice de Alfabetização Financeira & 211 & .33 & 1.0 & .6955 & 0.13729 \\
\hline
\end{tabular}

Fonte: Dados da pesquisa - SPSS-26 (2021).

Os índices associados a cada um dos construtos são considerados baixos, entretanto verifica-se que o conhecimento financeiro (educação financeira) foi o que apresentou maior fragilidade. Em que pese os resultados encontrados, nesta pesquisa, referirem-se aos discentes do interior do Ceará e originários dos países lusófonos; outros estudos nacionais e estrangeiros encontraram também baixos resultados para o conhecimento financeiro, sinalizando uma necessidade de priorização para esse tipo de conhecimento.

A melhoria dos índices alfabetização financeira poderá ocorrer através de ações e programas que modifiquem o comportamento, atitude e o conhecimento da população, nesse sentido registram-se duas experiências. Em relação ao esforço realizado pelo Banco Central para melhorar os níveis de educação financeira instituiu o Programa de Educação Financeira (PEF-BC), Matta (2007) ao avaliar o programa junto a 590 universitários do Distrito Federal, encontrou que a oferta e a demanda estavam alinhadas quanto aos tipos de fontes e gratuidade, mas consideraram que as fontes não eram de fácil acesso, apresentou alinhamento em termos das Finanças Pessoais, mas relativo em relação à Utilidade de Crédito e parcial, no que se refere à Gestão Financeira Pessoal.

Há de salientar que esforços foram envidados e tiveram relativo sucesso, ENEF (2012) apresentou os resultados do projeto piloto de educação financeira junto aos alunos do ensino médio, no período de 2010 até dezembro de 2011. O estudo utilizou os dados do Banco Mundial, realizaram uma avaliação de impacto do projeto, através do método de atribuição aleatória e uma avaliação aleatória e encontraram que o programa de educação financeira aumentou o conhecimento financeiro dos alunos, assim como das suas atitudes; levando a que estejam mais propensos a poupar e administrar suas despesas, conversar com seus pais sobre questões financeiras e ajudar a organizar orçamento familiar. Entretanto, sabe-se que a mudança de comportamento e a educação é um processo contínuo, carecendo de avaliações permanentes, seja para confirmação do programa, quanto para possíveis correções.

A Tabela 2 apresenta as características da amostra dos universitários nacionais e estrangeiros de todos os cursos ofertados em 2018, dos Campi do Ceará. 
Tabela 2 - Perfil dos respondentes.

\begin{tabular}{|c|c|}
\hline Característica & $\%$ \\
\hline Faixa etária: $20-24$ anos & 62.9 \\
\hline Gênero: & \\
\hline a. Masculino & 54.0 \\
\hline b. Feminino & 44.5 \\
\hline c. Outro & 1.5 \\
\hline Estado civil - Solteiro & 91.5 \\
\hline Sem filhos & 91.1 \\
\hline Pagam aluguel & 60.6 \\
\hline Dividem a moradia (colegas ou estudantes) & 45.5 \\
\hline Adultos (de 18 anos ou mais) -mesmo local & 74.2 \\
\hline Nacionalidade: & \\
\hline a. Brasileira & 65.4 \\
\hline b. Brasileira (Quilombola, Indígena) & .9 \\
\hline c. Angola & 11.8 \\
\hline d. Guiné-Bissau & 19.9 \\
\hline e. Moçambique & 1.5 \\
\hline f. São Tomé e Príncipe & .5 \\
\hline Religião: & \\
\hline a. Católica & 48.8 \\
\hline b. Evangélica & 22.7 \\
\hline c. Não tem religião & 22.3 \\
\hline d. Islâmica & 1.4 \\
\hline $\begin{array}{l}\text { e. Outras(Candomblé,Espírita, Ubamda,Acreditam em Deus, } \\
\text { Muçulmano e Daime). }\end{array}$ & 4.8 \\
\hline Curso: & \\
\hline a. Administração Pública & 15.2 \\
\hline b. Agronomia & 10.0 \\
\hline c. Antropologia & .9 \\
\hline d. Bacharelado em Humanidades & 8.1 \\
\hline e. Ciências Biológicas & 10.4 \\
\hline f. Engenharia da Computação & 2.4 \\
\hline g. Engenharia de Energias & 4.7 \\
\hline h. Enfermagem & 9.5 \\
\hline i. Farmácia & 1.4 \\
\hline j. Física & 3.8 \\
\hline k. História & 4.7 \\
\hline 1. Letras-Inglês & 1.4 \\
\hline m. Letras-Português & 7.6 \\
\hline n. Matemática & 7.6 \\
\hline o. Pedagogia & 3.8 \\
\hline p. Química & 6.2 \\
\hline q. Sociologia & 2.4 \\
\hline Índice de Desempenho do Estudante (IDE) & \\
\hline a. $9-10$ & 13.3 \\
\hline b. $8.9-8$ & 37.9 \\
\hline c. $7.9-7.0$ & 32.7 \\
\hline d. $\quad 5.1-6.9$ & 15.2 \\
\hline e. Abaixo de 5.0 & 0.9 \\
\hline
\end{tabular}

Fonte: Dados da pesquisa - SPSS-26 (2021).

Com base na Tabela 2 construiu-se o perfil dos respondentes nacionais e estrangeiros, em que, na sua maioria são solteiros (as), estão na faixa etária entre 20 a 24 anos, não têm filhos, pagam aluguel, dividem a moradia com amigos, colegas ou estudantes, verificou-se um percentual equitativo entre o público masculino e feminino e assemelham-se aos resultados 
compilados na Tabela 17 do OECD/INFE (2016 $)$. A intitulação como outros (considerada toda e qualquer opção sexual), representou $1.4 \%$ da amostra. Apesar de existir a prevalência da religião católica, observou-se que o percentual para aqueles que se intitulam como sendo evangélicos e de que que não têm religião, apresentaram percentuais muito próximos. Entretanto, um dos aspectos mais significativos é o de que essa amostra corresponde aos universitários dos países lusófonos e, para além disso, contempla, no Brasil, uma comunidade até então extremamente excluída, que são aqueles que se intitulam como sendo quilombolas e os indígenas. Além disso, o estudo conseguiu obter respostas dos discentes de todos os cursos ofertados no período.

Explorando os resultados dos construtos e do índice de alfabetização financeira (OCDE, 2018) construíram algumas estatísticas descritivas que permitem caracteriza os resultados encontrados, conforme apresentado na Tabela 3.

Tabela 3 - Estatística descritiva dos construtos e do Índice de Alfabetização Financeira-OCDE.

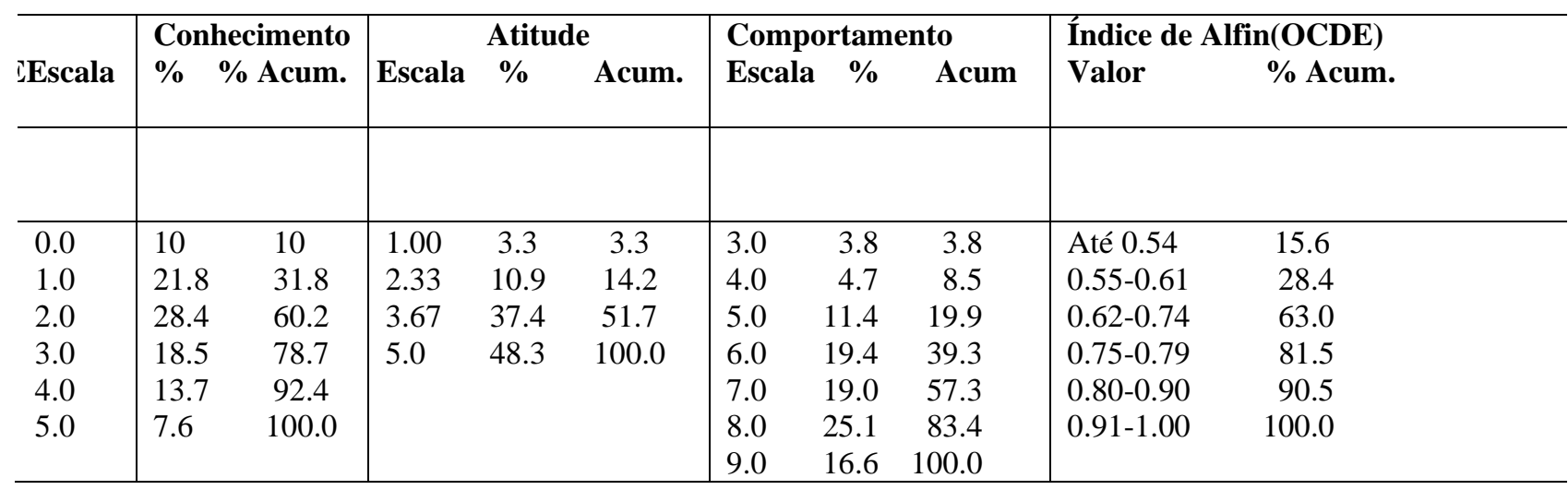

Fonte: Dados da pesquisa - SPSS-26 (2021).

Em termos do construto de conhecimento financeiro, observa-se que $60.2 \%$ da amostra apresentou um índice de 2.0; enquanto que, em termos de atitude financeira, $51.7 \%$ está na escala de pontuação de até 3.67. No que se refere ao comportamento financeiro, 57.3\% estão na escala de 7.0, considerando que o total na escala é de 9.0. Por fim, o índice de alfabetização financeira, considerando a metodologia OCDE (2018), 63\% encontram-se na faixa de 0.62 a 0.74 .

Considerando que o estudo não se ateve a tão somente calcular os construtos que iriam compor o índice de alfabetização financeira, com base na metodologia da OCDE (2018) e, com vistas a explorar a possível influência das variáveis socioeconômicas e demográficas sobre o grau de alfabetização, várias investigações e explorações estatísticas foram realizadas com vistas a investigar a existência de algum poder explicativo dessas variáveis, como descrito através da Equação 2.

Para o tratamento de dados utilizamos o modelo linear automático por considerar-se o mais indicado para o tipo de dados da amostra. Verificou-se, primeiramente, a normalidade através do Teste de Shapiro-Wilk, com p. > 0.05, assegurando assim a normalidade da distribuição dos resíduos. Para atestar da inexistência de autocorrelação de entre as variáveis inclusas no modelo estatístico, utilizou-se o teste de correlação de Pearson, conforme apresentado na Tabela 4. Verifica-se que nenhuma das variáveis está autocorrelacionada, daí não tendo efeitos nos resultados estatísticos.

\footnotetext{
${ }^{6}$ Pesquisa internacional contemplando alguns países em desenvolvimento e 17 países da OECD, totalizando 29 países; 51.650 adultos (18 a 79 anos), usando o Kit de Ferramentas da OECD para coletar dados comparáveis entre países, dado a unificação das questões. Salienta-se que o Brasil não figura na pesquisa OECD-INFE (2020).
} 
Tabela 4 - Alfabetização Financeira - Coeficientes de correlação - Método: Pearson.

\begin{tabular}{|c|c|c|c|c|c|c|c|c|c|}
\hline Construto/Questão & ALFNOR & Q4 & Q3 & Q5 & Q6 & Q7 & Q8 & Q10 & Q_25 \\
\hline ALFNOR & 1.00 & & & & & & & & \\
\hline Q4_Idade & -.080 & 1.00 & & & & & & & \\
\hline Q3_Gênero & -.030 & .078 & 1.00 & & & & & & \\
\hline Q5_Nacionalidade & -.091 & $.220 * *$ & .085 & 1.00 & & & & & \\
\hline Q6_Religião & -.082 & .100 & $.212 * *$ & .013 & 1.00 & & & & \\
\hline Q7_Estado_civil & -.004 & .054 & -.069 & -.056 & -.017 & 1.00 & & & \\
\hline Q8_Dependentes & .109 & $-.453 * *$ & $.152 *$ & $-.201 * *$ & .047 & $-.173 * *$ & 1.00 & & \\
\hline Q10_Curso & -.051 & $.121 *$ & -.033 & .029 & .069 & .107 & -.046 & 1.00 & \\
\hline Q25_IDE & $.117 *$ & -.081 & .040 & -.041 & .090 & $-.143^{*}$ & .106 & $-.121 *$ & 1.00 \\
\hline
\end{tabular}

* a correlação é significante ao nível de 0.05 ** a correlação é significante ao nível de 0.01. Fonte: Dados da pesquisa - SPSS-26 (2021).

Uma vez assegurado os pressupostos básicos para os cruzamentos estatísticos, utilizou-se, o Modelo Automático Linear para garantir a robustez dos resultados e a qualificação das variáveis independentes com maior efeito na variável dependente, conforme demonstrado na Figura 2.

Figura 2 - Modelo Automático Linear (LINEAR).

\section{Effects}

Target: ALFNOR

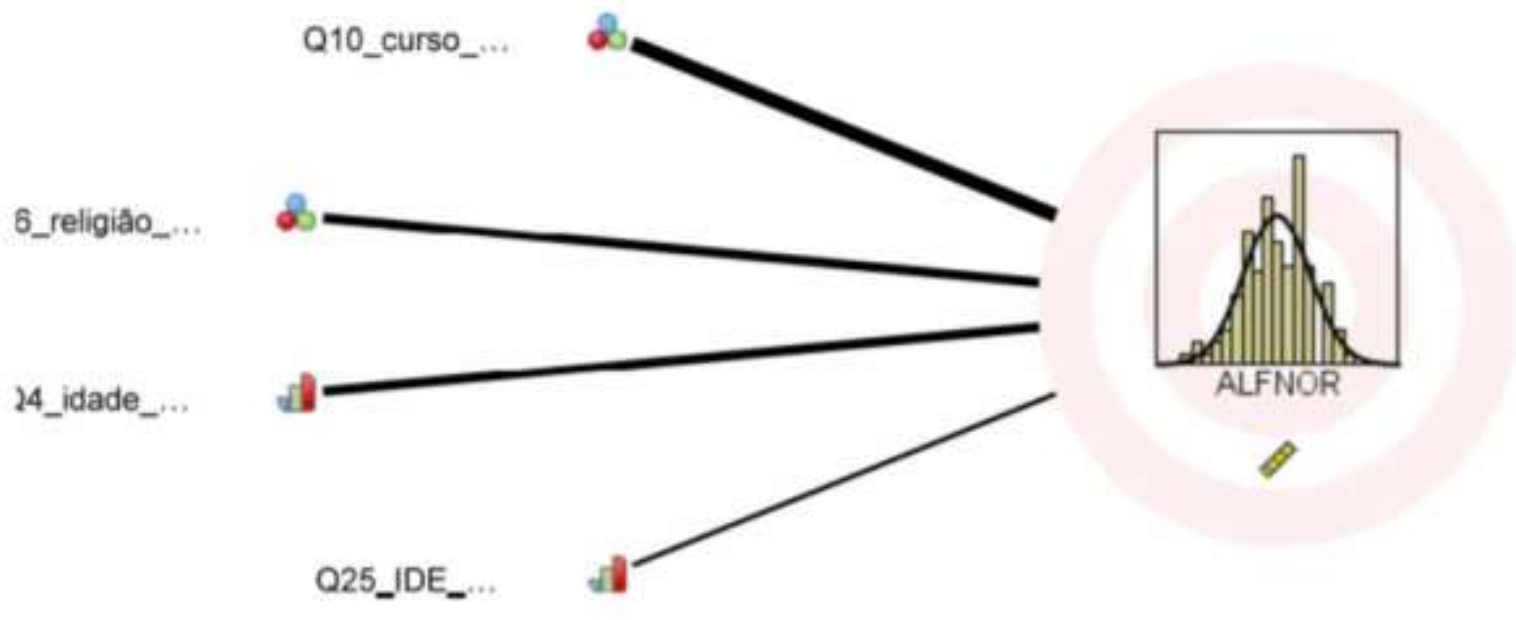

Fonte: Dados da pesquisa - SPSS-26 (2021).

Os resultados encontrados através do modelo evidenciam os efeitos do curso, da religião, da idade e do IDE sobre o índice de alfabetização financeira. Observa-se, pela Figura 1, que o curso foi o que apresentou maior efeito (F = 5,445 e Sig, $.001)$, em seguida a religião ( $F=4,696$ e Sig. .010, depois a idade (F=4,036 e Sig. .019) e, por fim o IDE. A Figura 3 apresenta os resultados em termos da estatística $\mathrm{F}$ e a significância. 
Figura 3 - Resultados dos efeitos do modelo- SPSS-26 (2021).

\section{Effects}

\section{Target: ALFNOR}

\begin{tabular}{|c|c|c|c|c|c|c|c|c|}
\hline Sou & reo & Sum of Squares & df & Mean Square & $\mathbf{F}$ & Sig. & Importa & ance \\
\hline Cor & rected Model $\boldsymbol{\nabla}$ & 0,658 & 8 & 0,082 & 5,036 & .000 & & \\
\hline Q10 & curso_transformed & 0.267 & $\mathbf{3}$ & 0,089 & 5,446 & .001 & o. & 463 \\
\hline Q6 & religiao_transformed & 0,153 & 2 & 0,077 & 4,696 & 010 & of & 261 \\
\hline Q4 & fiade_transformed & 0,132 & 2 & 0.066 & 4.036 & 019 & of & 224 \\
\hline Q25 & IDE_transformed & 0,037 & 1 & 0,037 & 2.25 .4 & .135 & of & 063 \\
\hline Res: & duat & 3,300 & 202 & 0,016 & & & & \\
\hline Cort & rected Total & 3.968 & 210 & & & & & \\
\hline
\end{tabular}

Fonte: Dados da pesquisa - SPSS-26 (2021).

Com vistas a identificar o curso que apresentou maior efeito sobre o índice de alfabetização financeira, através da Figura 4 pode-se observar que os cursos que apresentaram maiores e menores efeitos sobre o índice de alfabetização financeira.

Figura 4 - Média dos efeitos estimados dos cursos.

Target: ALFNOR

Estimated means charts for the top ten significant effects $(p<, 05)$ are displayed.

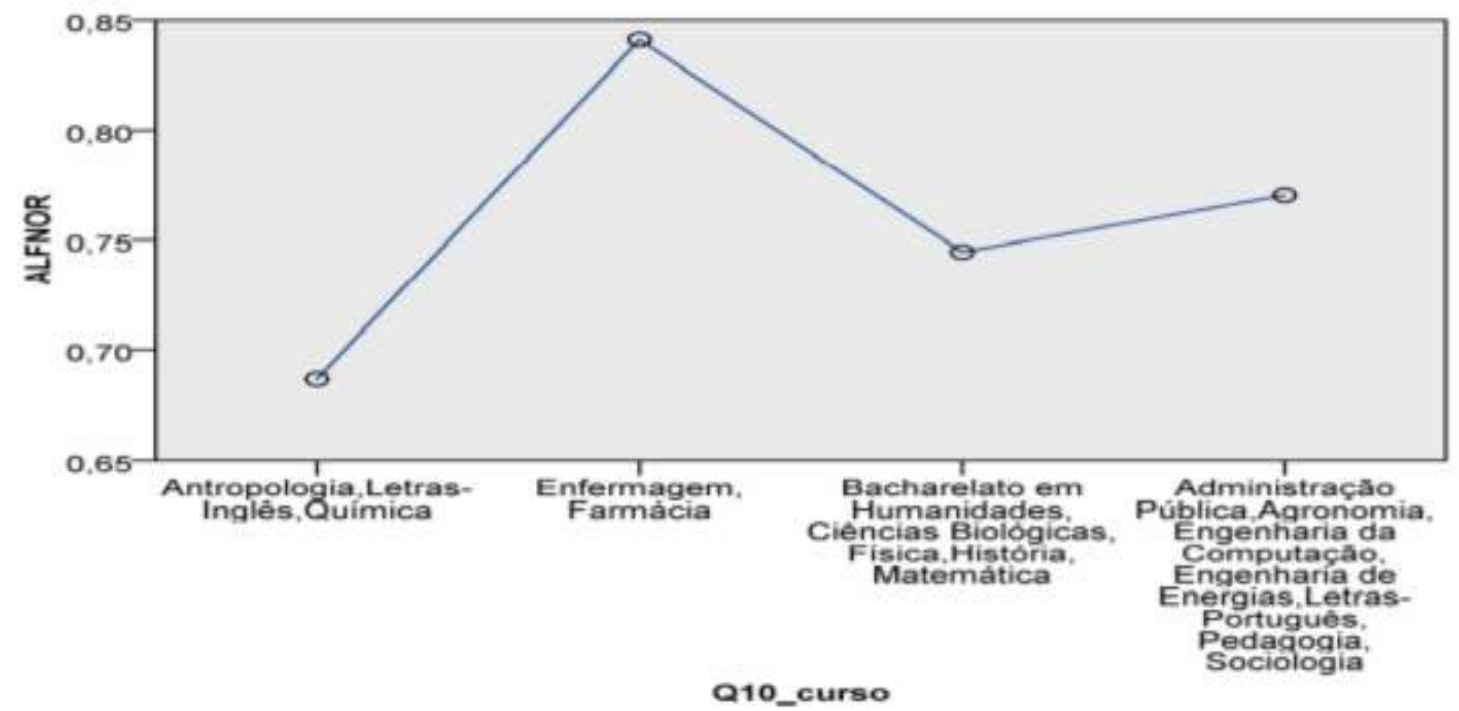

Fonte: Dados da pesquisa - SPSS-26 (2021).

Observa-se que os cursos que apresentaram os menores efeitos sobre o índice de alfabetização financeira foram os de Antropologia, Letras-inglês e Química; enquanto que os de Enfermagem e Farmácia apresentaram os maiores efeitos. A Figura 5 apresenta os efeitos da religião. 
Figura 5 - Média dos efeitos estimados da religião.

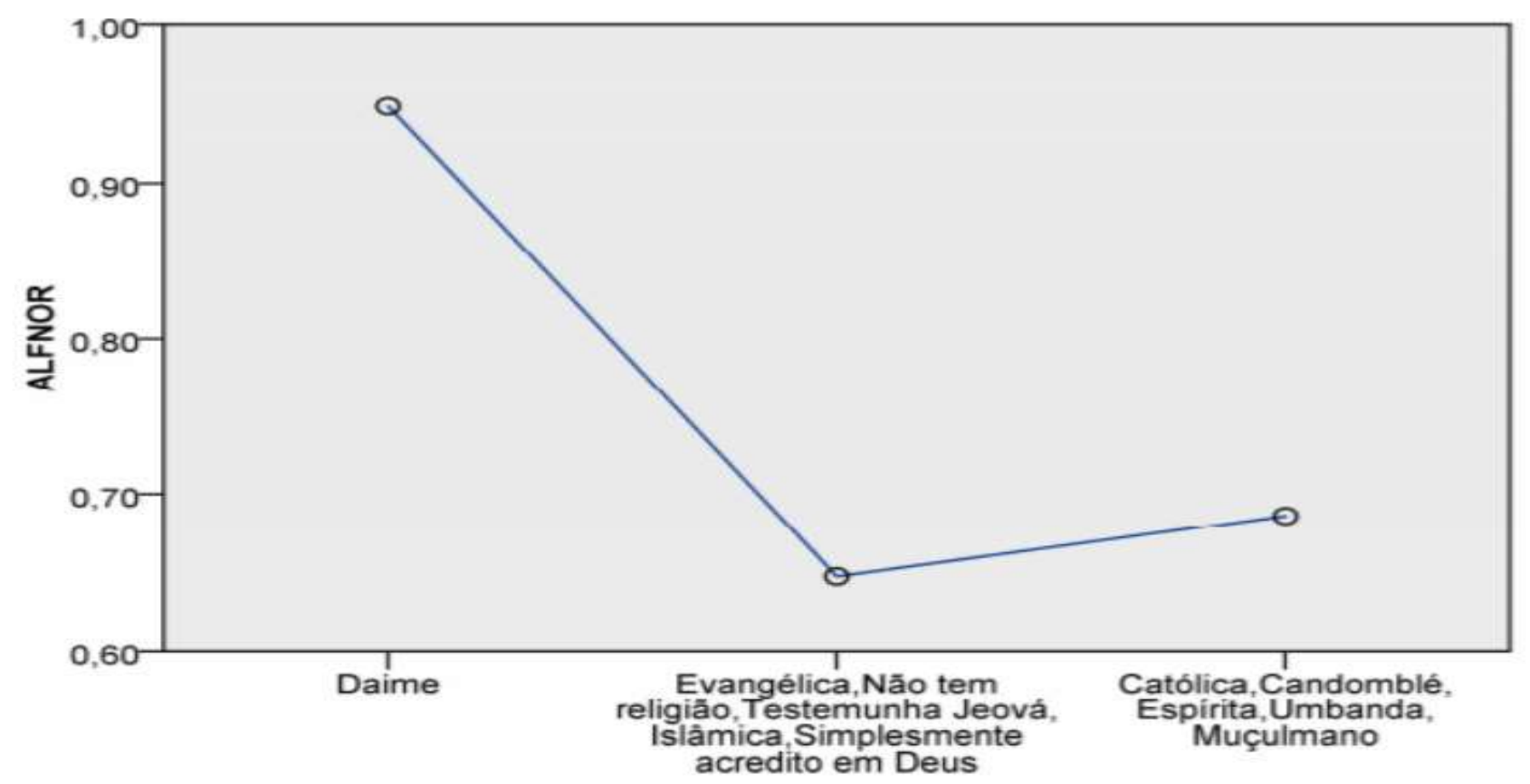

Fonte: Dados da pesquisa (SPSS-26, 2021).

Pode-se inferir, pela Figura 5, que os maiores índices de alfabetização financeira foram encontrados dentre os discentes que têm como religião o Daime, seguido pelo grupo de religião encabeçado pela Católica e o outro grupo pela Evangélica.

\section{Considerações Finais}

O grau de alfabetização financeira dos universitários dos países lusófonos dos Campi do Ceará, da Unilab não foi considerado satisfatório, mas não diverge, em muito, dos estudos realizados com outros universitários, em outras regiões brasileiras.

No estudo da OECD/INFE (2016) o baixo índice de alfabetização está vinculado a diferentes construtos, em alguns casos, atribuídos ao conhecimento financeiro (Brasil e de outros países) e em outros casos, ao comportamento financeiro (Latvia, Estônia, Polônia e Croácia).

Esse estudo confirma a avaliação da OECD/INFE (2016), dado que o construto de conhecimento financeiro apresentou maior fragilidade (0.237) e distancia-se daquele reportado pelos resultados para o Brasil, realizados pela OECD/INFE (2016, p. 8), em que pontuação obtida foi de 0.43. Entretanto, ressalta-se que o percentual dos respondentes que acertaram todas as questões no estudo da OECD/INFE (2016, p.66) são aproximadamente os mesmos (8\% para a OECD/INFE, 2016) e aquele apresentado na tabela $3(7.6 \%)$.

Enseja-se, por conseguinte, uma atenção especial tantos para os gestores quanto para os formuladores de políticas públicas dos países lusófonos estudados, para o desenho de ações e programas que visem melhorar as atitudes e comportamentos financeiros; em especial para os conhecimentos e as práticas financeiras dos discentes, ressaltando que programas continuados são mais efetivos no curto e no longo prazo, conforme evidências encontradas por Lusardi, Michaud \& Mitchell (2018).

Ademais, sugere-se em estudos futuros sobre a Alfabetização Financeira a exploração de outras variáveis explicativas, bem como de outros públicos-alvo e dos programas ofertados. 


\section{Referências}

Ansong, A. (2011). Level of knowledge in personal finance by university freshmen business students. African Journal of Business Management, 5(22), 89338940. http://www.academicjournals.org/ajbm/pdf/pdf2011/30Sept/Ansong.pdf

Atkinson, A. \& Messy, F. (2012). Measuring Financial Literacy: Results of the OECD / International Network on Financial Education (INFE) Pilot Study , OECD Working Papers on Finance, Insurance and Private Pensions, no. 15, OECD Publishing. http://dx.doi.org/10.1787/5k9csfs90fr4-en.

Agarwalla, S. K.; Barua, S.; Jacob, J. \& Varma, J. R. (2015). Financial Literacy among Working Young in Urban India. World Development, 67, 101-109. https://doi.org/10.1016/j.worlddev.2014.10.004

Barros, A. S. X. (2015). Expansão da educação superior no Brasil: limites e possibilidades. Educação \& Sociedade. Revista de Educação de Ciência da Educação. https://doi.org/10.1590/ES0101-7330201596208. https://www.scielo.br/j/es/a/NGJT56L Bxz9VCDCp7gr86Tf/?lang=pt.

Bernheim, B. D.; Garrett, D. M. \& Maki, D. M. Education and saving: the long term effects of high school financial curriculum mandates. National Bureau of Economic Research. 10.3386/w6085. https://www.nber.org/papers/w6085.

Borges, B. R. \& Botelho, D. R. (2020). Uma década de pesquisa em Alfabetização e Educação Financeiras: um estudo bibliométrico. XVII Congresso USP de Iniciação Científica em Contabilidade. São Paulo. 20 a 31 julho de 2020.

Brasil (2010) Lei 12.289, de 20 de julho de 2010. http://www.planalto.gov.br/ccivil_03/_Ato2007-2010/2010/Lei/L12289.htm

Brasil (2020). Decreto 10.393, de 09 de junho de 2020. https://legis.senado.gov.br/norma/32247511/publicacao/32247759.

Cenci, J. J., Pereira, I \& Barichello, R. (2015). Educação financeira, planejamento familiar e orçamento doméstico: um estudo de caso. Tecnológica-Revista Científica. https://uceff.edu.br/revista/index.php/revista/article/view/61.

ENEF(2010). Brasil: Implementando a Estratégia Nacional de Educação Financeira. https://www.bcb.gov.br/pre/pef/port/Estrategia_Nacional_ Educacao_Financeira_ENEF.pdf.

ENEF (2012). Resultados da avaliação de impacto do projeto piloto de educação financeira nas escolas. Release elaborado pela BM\&FBOVESPA baseado nos dados informados pelo Banco Mundial, para o $2^{\circ}$. Workshop de Divulgação dos Resultados da ENEF-Estratégia Nacional de Educação Financeira, em junho de 2012. https://www.vidaedinheiro.gov.br/wp-content/uploads/2017/04/avaliacao_educacao_financeira_escolas.pdf.

Gujarati, D. (2011). Econometria básica (5a ed). Mc Graw Hill.

Huston, S. J. (2010). Measuring financial literacy. The Journal of Consumer Affairs, http://onlinelibrary.wiley.com/doi/10.1111/j.17456606.2010.01170.x/pdf.

Holzmann, R.\& Miralles, M. P. (2005). The role, limits of, and alternatives to financial education in support of retirement saving in the OECD, Eastern Europe and beyond. The World Bank, out/, 2005. http://info.worldbank.org/etools/library/view_p.asp?205715

Hung, A. A., Parker, A. M., \& Yoong, J. (2009). Defining and measuring financial literacy.. SSRN Electronic Journal 708(708). 10.2139/ssrn.1498674. January 2009.

Jacob, K., Hudson, S. \& Bush, M. (2000). Tools for survival: An analysis of financial literacy programs for lowerincome families. Woodstock Institute. http://www.woodstockinst.org/research/tools-survivalanalysis-financial-literacy-programs-lower-income-families.

Jobim, S. S. A. \& Losekann, V. L. (2015) Alfabetização Financeira: mensuração do comportamento e conhecimento financeiros dos universitários da Universidade da Região da Campanha, Rio Grande Do Sul. Revista Sociais e Humanas, 10.5902/231717818835.

Lakatos, E.M. \& Marconi, M de A. (2021) Fundamentos de Metodologia Científica. (9a ed.). Atlas.

Lima, R.S. L., Barbalho, P. R. M., Nascimento, H. L., Medeiros Neto, J. S. \& Brito, M. L. de A. (2019). O estudo das finanças pessoais no âmbito universitário. Research, Society and Development, 8(2), 1-10.

Lucci, C. R., Zerrenner, S. A., Verrone, M. A. G., \& Santos, S. C. (2006, agosto). A Influência da Educação Financeira nas Decisões de Consumo e Investimento dos Indivíduos. Anais do SEMEAD - Seminários em Administração, São Paulo, SP, Brasil, 09.

Lusardi, A. \& Mitchell, O. S. (2005). Financial literacy and planning: implications for retirement wellbeing. [Working Paper No 108]. Michigan Retirement Research Center, University of Michigan. http://deepblue.lib.umich.edu/bitstream/handle/2027.42/49432/wp108.pdf?

Lusardi, A., \& Mitchell, O. S. (2011). Financial literacy and retirement planning in the United States. Journal of Pension Economics and Finance, Cambridge University Press, 10(04), 509-525. http://dx.doi.org/10.1017/S147474721100045X. http://www.nber.org/papers/w17078.

Lusardi, A., Mitchell, O. S. \& Curto, V. (2010). Financial literacy among the young. The Journal of Consumer Affairs, 44(02), 358-380. http://onlinelibrary.wiley.com/doi/10.1111/j.1745-6606.2010.01173.x/pdf

Lusardi, A., Tufano, P. (2009). Debt literacy, financial experiences, and overindebtedness. [Working Paper $\mathrm{N}^{\mathrm{o}}$ 14808]. National Bureau of Economic Research, Cambridge, MA. http://www.nber.org/papers/w 14808.pdf?

Lusardi, Michaud \& Mitchell (2018). Assessing the impact of financial education programs: a quantitative model. Pension Research Council Working Paper. https://pensionresearchcouncil.wharton.upenn.edu/wp-content/uploads/2018/04/WP-2018-4-Lusardi-et-al.pdf 
Matta, R. C. B. (2007). Oferta e demanda de informação financeira pessoal: o Programa de Educação Financeira do Banco Central do Brasil e os universitários do Distrito Federal. [Dissertação de Mestrado, Universidade de Brasília, Brasília, Brasil]. Repositório Digital da Universidade de Brasília. https://repositorio.unb.br/handle/10482/5293

Menezhetti Neto, A., Falcetta, F. P., Rassier, L. H., \& Marchionatti, W. (2014). Educação Financeira. Edipucrs.

OECD (1960). https://www.oecd.org/general/conventionontheorganisationforeconomicco-operationanddevelopment.htm.

OECD (1990). Brasil como país parceiro. https://www.oecd.org/global-relations/keypartners/\#d.en.194387.

OECD (2005). Improving Financial Literacy: Analysis of Issues and Policies. OECD

https://www.oecd.org/finance/financial-education/improvingfinancialliteracyanalysisofissuesandpolicies.htm

OECD/INFE (2005). Recommendation of the Council on Financial Literacy. OECD http www.oecd.org/finance /financialeducation/35108560.pdf.https://legalinstruments.oecd.org/fr/instruments/OECD-LEGAL-0461.

OECD (2008). OECD. https://www.oecd.org/financial/education/oecd-international-network-on-financial-education.htm

OECD (2009). Improving financial literacy: analysis of issues and policies. OECD Centre: Paris, France. https://search.oecd.org/daf/fin/financialmarkets/improvingfinancialliteracyanalysisofissuesandpolicies.htm

OECD (2010). Education at a Glance. OECD Indicators, Publication 7/9/2010. https://www.oecd.org/education/skills-beyondschool/educationataglance2010indicators.htm

OECD. (2011). Measuring Financial Literacy: Core Questionnaire in Measuring Financial Literacy, Questionnaire and Guidance Notes for conducting an Internationally Comparable Survey of Financial literacy. OECD Centre: Paris, France.

OECD (2013). Advancing National Strategies for financial education. OECD. https://www.oecd.org/finance/financialeducation/G20_OECD_NSFinancialEducation.pdf

OECD. (2013). PISA 2012 - Assessment and Analytical Framework. Paris: OECD, 2013. https://www.oecd.org/pisa/pisaproducts/PISA\%202012\%20frame work\% 20e-book_final.pdf

OECD/INFE (2016). International Survey of Adult Financial Literacy Competencies. OECD/INFE (12 oct 2016). https://www.oecd.org/finance/oecdfinancial-literacy-study-finds-many-adults-struggle-with -money-matters.htm.

OECD/INFE (2015). Toolkit for measuring financial literacy and financial inclusion. OECD/INFE (march, 2015). https://www.oecd.org/daf/fin/financialeducation/2015_OECD_INFE_Toolkit_Measuring_Financial_Literacy.pdf

OECD/INFE (2016). Financial literacy study finds many adults struggle with money matters. OECD/INFE (12oct,2016).https://www.oecd.org/financial/education/oecd-infe-survey-adult-financial-literacy-competencies.html.

OECD/INFE (2018). Toolkit. for measuring financial literacy and financial inclusion. OECD/INFE (may, 2018).https://www.oecd.org/finance/financialducation/ES_2018\%20OECD\%20INFE\%20Toolkit.pdf

OECD/INFE (2020). International survey of adult financial literacy. OECD/INFE (25 june, 2020).

https://www.oecd.org/financial/education/launchoftheoecdinfeglobalfinancialliteracysurveyreport.htm.

Poltrich, A. C. G., Vieira, K. M. e Ceretta, P. S. (2013). Nível de alfabetização financeira dos estudantes universitários: afinal, o que é relevante? Revista Eletrônica de Ciência Administrativa (RECADM). https://doi.org/10.5329/RECADM.2013025. http://www.periodicosibepes.org.br/index.php/recad $\mathrm{m} /$ article/view/1656.

Poltrich, Vieira e Kirch (2014). Você é alfabetizado financeiramente? Descubra no termômetro de alfabetização financeira. FGV Repositório Digital. https://hdl.handle.net/10438/18826. https://bibliotecadigital.fgv.br/dspace/handle/10438/18826.

Potrich, A. C. G. Vieira, K. M. , \& Kirch, G. (2015). Determinantes da Alfabetização Financeira: Análise da Influência de Variáveis Socioeconômicas e Demográficas. Revista Contabilidade \& Finanças, 26(69), 362. https://doi.org/10.1590/1808-057x201501040

Poltrich. A. C. G. Vieira,K. M. V. e Paraboni, A. L.. (2013). O que influencia a alfabetização financeira dos estudantes universitários. XII Seminários em Administração, 2013.

Reis, L. C., Borgis, M. O. \& Silva, V. G. M. (2021). Educação Financeira: Aposentadoria sob a ótica dos acadêmicos de uma universidade pública do centro oeste de Minas Gerais. Research, Society and Development, 10 (1), 1-15.

Remund, D. L. (2010). Financial literacy explicated: the case for a clearer definition in an increasingly complex economy. The Journal of Consumer Affairs, 44(02), 276-295. http://onlinelibrary.wiley.com/doi/10.1111/j.1745-6606.2010.01169.x/pdf

Santos, R. A. T., Rodrigues, W. \& Nunes, C. de O. (2021). Os efeitos da educação financeira no comportamento de consumo: Um estudo com idosos de baixa renda. Research, Society and Development, 10 (5), 1-11.

Sekita, S. (2011). Financial literacy and retirement planning in Japan. Journal of Pension Economics and Finance, 10(04), 637-656.

Seixas, G. S., Santarosa, M. C. P. \& Ferrão, N. S. (2020). Educação Financeira na EJA: proposta de uma sequência didática à luz da Teoria da Aprendizagem Significativa Crítica. Research, Society and Development, 9(11), 1-23. 
Research, Society and Development, v. 10, n. 9, e20210917527, 2021

(CC BY 4.0) | ISSN 2525-3409 | DOI: http://dx.doi.org/10.33448/rsd-v10i9.17527

Severino, A. J. (2017). Metodologia do trabalho científico. (24a ed.) Cortez.

Silva, E. A. da. (2016). Alfabetização Financeira: reflexões a partir de um estudo de caso com idosos na Irlanda. Mariana-MG, 2016. [Monografia, Bacharel em Administração, Departamento de Ciências Administrativas, Instituto de Ciências Sociais Aplicadas, Universidade Federal de Ouro Preto, Mariana]. Biblioteca Digital de TCCs. https://www.monografias.ufop.br/handle/35400000/571Mariana, 2016.

Silva, C. C, Rodrigues, M. P. S., Moura, J. A. \& Castro, W. A. (2019). Educação financeira: um estudo envolvendo os alunos de uma instituição de ensino superior da cidade de Divinópolis em Minas Gerais. Research, Society and Development, 8(8),1-22. e07881177.

Vieira, K. M., Moreira Júnior, F. de J., Potrich, A. C. G. (2019). Indicador de educação financeira: proposição de um instrumento a partir da teoria da resposta ao item. Educação \& Sociedade, 40. https://doi.org/10.1590/ES0101-73302018182568.

Yang, H. (2013). The case for being Automatic:Introducing the Automatic Linear Modelling (LINEAR). General Linear Model Journal, 39 (2). http://www.glmj.org./archives/_GLMJ_201439n2.html..

Zuliani, A. L. B., Flores, S. A. M., Floriano, M. D. P., Josende, A. C. \& Ramos, T. J. F. (2020). Os jovens militares são alfabetizados financeiramente? Estudo em uma organização da fronteira gaúcha. Unichristus - Revista Gestão em Análise - ReGea. 10.12662/2359-618xregea.v9i1.p13653.2020.https://periodicos.unichristus.edu.br/gestao/article 